\title{
An object-oriented simulation of anorexia based on brain structure interaction
}

\author{
MICHELLE A. PETHERICK, DAVID V. REYNOLDS, and MARC SEGUIN \\ University of Windsor, Windsor, Ontario, Canada \\ and \\ KARL MIKES \\ Computer Systems Resources, Windsor, Ontario, Canada
}

\begin{abstract}
A computer simulation using an object-oriented strategy was developed to model brain mechanisms that underlie eating disorders. The simulation was based on neuroanatomical and neurochemical evidence from published literature. Testing of the simulation required that the activity levels of the raphe nuclei of the brain stem be altered and the resulting activity levels of associated brain structures be compared with results from human and animal data. The results supported the serotonin hypothesis of anorexia that increasing the activity level of the raphe nuclei would result in dramatic changes in the activity level of the paraventricular nucleus. The findings are discussed with respect to the feasibility of computer simulations to test theories of brain-based disorders.
\end{abstract}

Traditionally, physiological psychologists propose theories and carry out experiments to test them; results from these experiments are then analyzed to determine if they contradict or confirm the theories. The value of this experimental approach has been amply demonstrated. Alternatively, computer simulations have been used to test theories, but less often. The current availability of computers and the development of appropriate computer languages have made computer-based simulations increasingly feasible. The use of the object-oriented approach to simulate the neurochemical basis of anorexia nervosa is described in the present study.

Eating disorders, such as anorexia nervosa, are reported to be widespread, and considerable research is being devoted to understanding the brain bases of these disorders. Anorexia is characterized by severely depressed eating behavior, associated with medical complications (Jimerson, Lesem, Hegg, \& Brewerton, 1990). Physiological psychologists assume that the abnormal behaviors exhibited in anorexia are symptoms of changes in brain function and that neurochemical abnormalities may be a contributor. Considerable research has suggested that altered serotonin (5-HT) activity is a key element in pathological eating behavior (Goldbloom, Hicks, \& Garfinkel, 1990a; Kaye, Gwirtsman, George, \& Ebert, 1991). Numerous animal studies have demonstrated that 5-HT promotes satiety and thus influences food intake (Aoyagi, Oomura, \& Shimizu, 1992). Additional investigations have reported altered 5-HT activity in eating-disorder patients (Goldbloom, Hicks, \& Gar-

Correspondence should be addressed to D. V. Reynolds, Department of Psychology, University of Windsor, Windsor, ON, Canada N9B 3P4. finkel, 1990b; Kaye et al., 1991). Pharmacological studies have also suggested that serotonergic systems are important in the regulation of feeding behavior. Specifically, serotonin antagonists, such as cyproheptadine, have been shown to enhance weight gain in anorexic patients and to increase binge and purge eating in bulimic patients (Walsh \& Devlin, 1992). Moreover, although serotonin agonists such as fluoxetine have been used in the treatment of bulimia to effectively reduce meal size, anorexic symptoms have manifested in some patients (Oliveros, Iruela, Caballero, \& Baca, 1992). On the basis of this evidence, the serotonin hypothesis of anorexia suggests that anorexia is the result of functional overactivity of 5-HT (Kaye, Ebert, Gwirtsman, \& Weiss, 1984).

Although various therapeutic strategies for anorexia have been developed, the significant mortality rate associated with this disorder, coupled with its increasing prevalence and high rate of relapse, suggests that effective treatment methods and valid research models are urgently needed. The present study developed a computer simulation of brain changes that may underlie the abnormal eating behavior exhibited in anorexia. Computer simulations require explicit representation. In their overview, Whicker and Sigelman (1991) state:

A flow chart, often developed prior to computer simulation, is a type of schematic model... the model itself typically is a representation of a structure, while the simulation is a representation of the structure in action... dynamic, unfolding over time between certain starting and ending points. (p. 5)

In our case, the flow chart is a drawing of known connections among brain structures, and hypothesized excitation or inhibition of other brain structures to which 
they project. This information is entered into the computer-simulation program, and the results are displayed. In this way, the simulation models the outcome of the dynamic interaction of these brain structures.

We have chosen to use an object-oriented approach in developing our simulations. This strategy for simulating complex systems embodies certain advantages, including rapid prototyping, extendibility, and flexibility. Because of these and other advantages, simulations can be changed as new information becomes available. A detailed discussion of the advantages of this approach is tangential here, but may be found elsewhere (Booch, 1991; Love, 1993; Reynolds \& Del Dotto, 1991).

We have developed a simple simulation development tool, or "shell," that can be used to model the interaction of brain structures. This simulation program, developed for PC-compatible microcomputers using MS DOS 6.1, was written in the object-oriented language Borland $\mathrm{C}++$ under Windows. The interface screen presents a series of menus, and the simulation is run using a computer mouse. The results of the simulation are displayed both numerically and graphically.

Before a brain system can be simulated, it must be sufficiently explicit to display as a flow chart showing brain structures projecting excitation or inhibition. A simple model of brain structures that affect eating is presented in Figure 1 (modified from Blundell, 1991).

The hypothalamus is considered an important central site of 5-HT's effect on eating behavior (Aoyagi et al., 1992). A number of serotonin receptors have been clearly mapped within the hypothalamus (Pazos, Probst, \& Palacios, 1987), and the suppressive effects of 5-HT in the paraventricular nucleus (PVN) have been well documented. Research has shown that PVN lesions reduce 5-HT-induced anorexia (Morley \& Blundell, 1988) and that direct infusion of 5-HT into the PVN leads to decreased feeding behavior (Aoyagi et al., 1992).

Although Anand and Brobeck (cited in Carlson, 1991) found that lesions of the lateral hypothalamic area (LHA) increased eating, the effects of 5-HT on LHA are

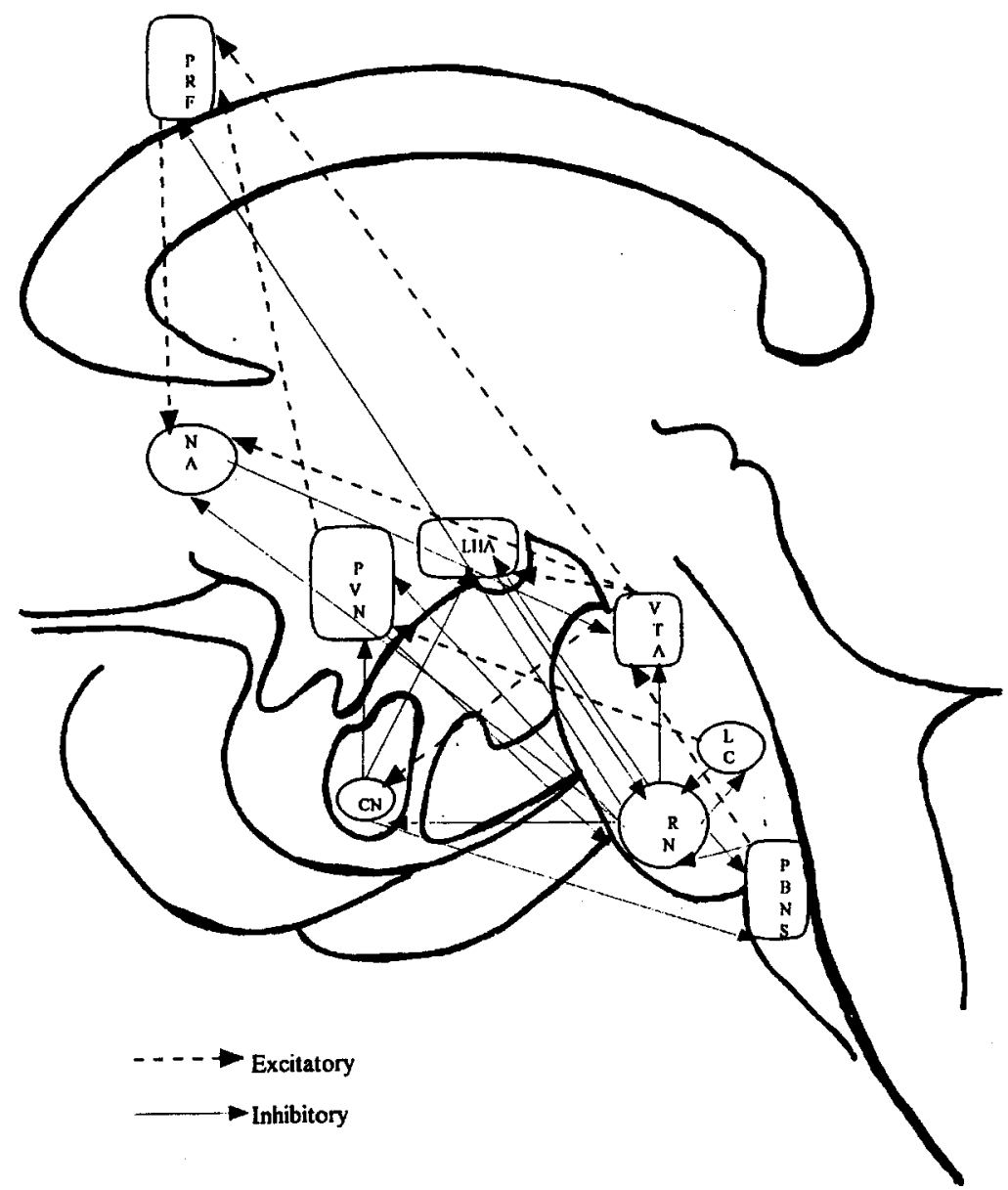

Figure 1. Interaction among associated brain structures in the serotonergic system implicated in the etiology of anorexia nervosa. $\mathrm{CN}=$ central nucleus of the amygdala; $\mathrm{RN}=$ raphe nuclei; $\mathrm{PBNS}=$ parabrachial nucleus and nucleus solitarius; $\mathrm{LC}=$ locus coeruleus; VTA = ventral tegmental area; LHA = lateral hypothalamic area; $P V N=$ paraventricular nucleus; NA = nucleus accumbens; PRF = prefinntal area. 
not clear. The central nucleus of the amygdala $(\mathrm{CN})$ is assumed to mediate conditioned emotional responses and seems to play an important part in physiological and behavioral responses to biologically significant events, including eating (Carlson, 1991).

The parabrachial nucleus (PB) and nucleus solitarius (NS) of the brain stem receive nutrient-related information and gustatory information from other parts of the body, and changes in their activity have an important effect on eating behavior (Blundell, 1991). The locus coeruleus (LC) is the primary source of neurons that deliver norepinephrine (NE) to the PVN. When directly injected into the PVN, NE leads to increased eating (Fava, Copeland, Schweiger, \& Herzog, 1989). Thus, it is reasonable to assume that the degree of eating behavior at a given time is at least partially related to the activity of $\mathrm{NE}$ from the LC and 5-HT from the raphe nuclei.

The serotonin hypothesis, as interpreted in light of the neuroanatomical evidence presented, would suggest that increased inhibition from the raphe nuclei would have a dramatic inhibitory influence on the PVN. To test this hypothesis, the activity level of the raphe nuclei in our simulation of the serotonergic system was increased to $125 \%$ and $150 \%$ in succession without reset. It was expected that the activity level of the PVN would decrease as a result of the increased inhibitory influence from the raphe nuclei.

\section{METHOD}

Neurochemical and neuroanatomical data were incorporated into a computer program using object-oriented methodology. A 486sx PC-compatible microcomputer was used to develop and run the serotonin simulation program, which was written in $\mathrm{C}++$. In order to avoid negative values in simulated brain structure activity (projected inhibition provides this function), all structures are preset to "bottom out" at $1 \%$ activity.

Brain diagrams based on published research were drawn to model the interaction of major structures and neurochemical projections of the serotonergic system and associated brain structures. These diagrams were then simplified to permit modeling by the computer program. A prototype of the serotonergic eating system was developed using the data provided in the figures. Initially, the functional levels of all the structures in the model were set at $100 \%$ to reflect normal functioning. Next, different activity levels were assigned different structures in the model to represent "normal" fluctuations (i.e., 90\%, 110\%, 100\%). The resulting activity levels in the remaining structures were then observed to see if they reacted so that the system tended to return to normal $(100 \%)$ activity.

After the simulation showed that it could function normally, experiments were carried out. The activity levels of one or more brain structures were changed, the simulation was run, and changes in activity levels of the remaining structures were observed. The changes in the activity of simulated brain structures were then compared with those obtained from animal and human data (Aoyagi et al., 1992; Fava et al., 1989; Jimerson et al., 1990).

In our experiments, the activity level of the raphe nuclei, which are the major originating structures of $5-\mathrm{HT}$, were increased to $125 \%$ and $150 \%$ in succession without reset. The resulting changes in the other brain structure's activity levels were then recorded. Possible treatments were then tested using the simulation.

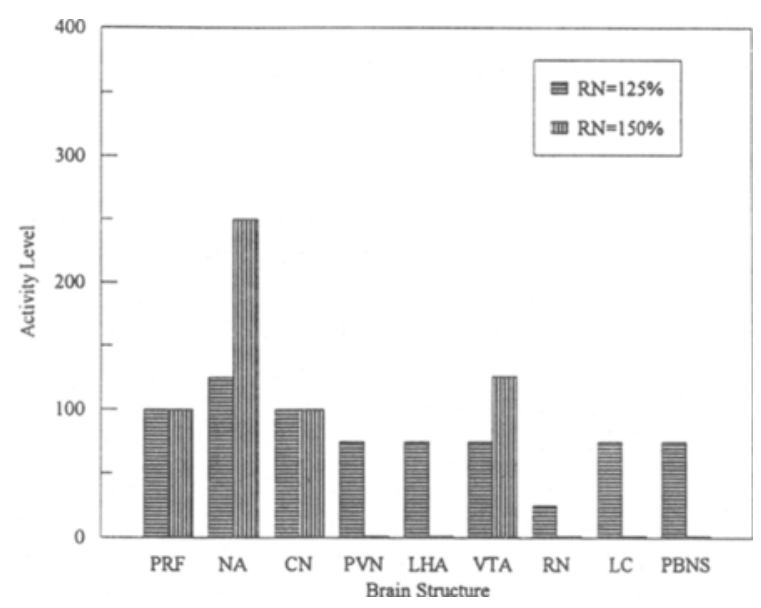

Figure 2. Activity levels of brain structures in the anorexia simulation, with 100 being normal, as a function of successive changes in raphe nuclei functioning to $125 \%$ and $150 \%$.

\section{RESULTS}

\section{Normalization}

The normalization test demonstrated that the simulation was relatively stable - that is, most of the brain structure's activity levels remained at or near $100 \%$ during minor fluctuations in the activity level of other brain structures. A few major fluctuations were observed, however, in the PVN and raphe nuclei, suggesting that further modification of the system may be necessary.

\section{Running of Simulation}

Increasing raphe nuclei activity by $25 \%$ led to a $25 \%$ reduction in PVN activity. Increasing the activity level of the raphe nuclei by an additional $25 \%$ led to a decrease of $99 \%$ in the PVN (see Figure 2).

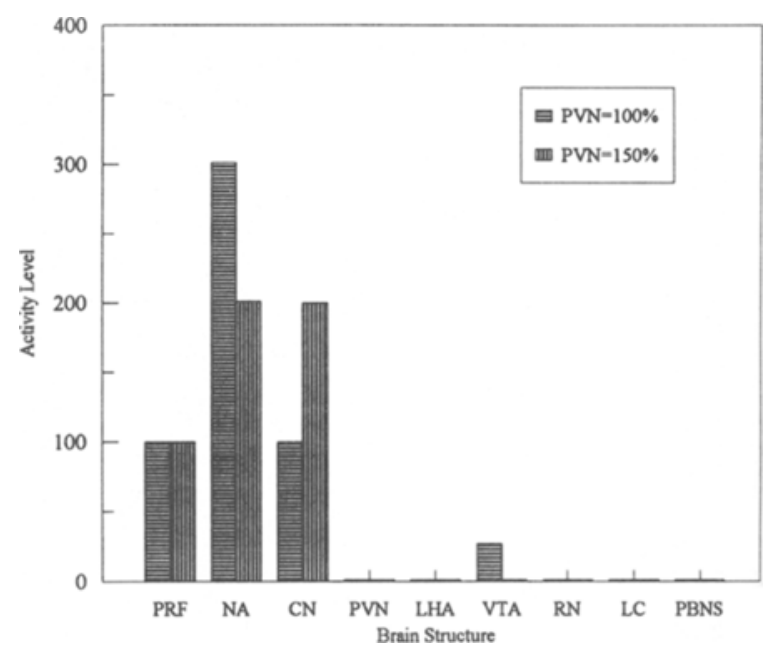

Figure 3. Activity levels of brain structures in the anorexia simulation, with 100 being normal, following changes in raphe nuclei functioning to $125 \%$ and $150 \%$ as a function of successive changes in PVN functioning to $100 \%$ and $150 \%$. 


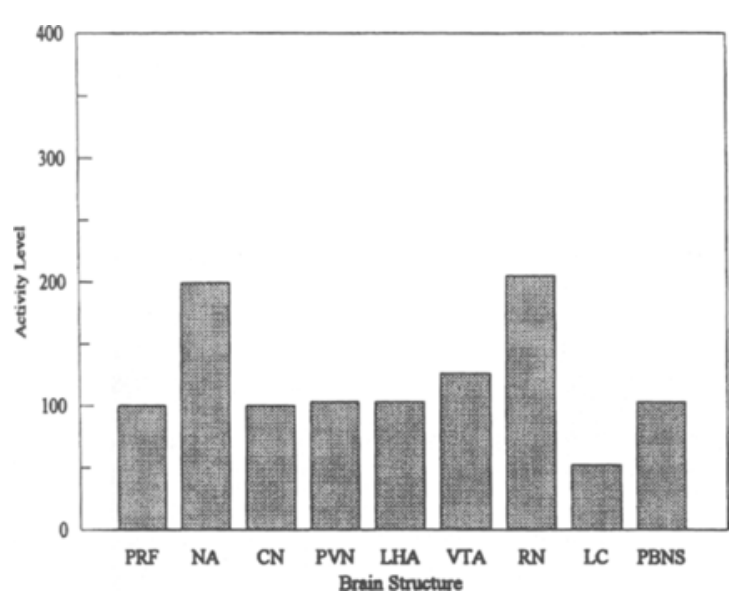

Figure 4. Activity levels of brain structures in the anorexia simulation, with 100 being normal, following changes in raphe nuclei functioning to $125 \%$ and $150 \%$ as a function of a change in VTA functioning to $75 \%$.

When simulating a possible treatment approach, we attempted to alter the activity level of PVN directly by increasing it to $100 \%$ and $150 \%$. However, because of its dynamic positive and negative feedback connections, PVN's activity level kept returning to $1 \%$, despite these drastic alterations (see Figure 3).

When the ventral tegmental area (VTA) was reduced to $75 \%$, however, PVN activity level returned to close to normal functioning or $103 \%$ (see Figure 4).

\section{DISCUSSION}

The normalization test demonstrated that the simulation interacted as a relatively stable system-that is, wide fluctuations in brain structure activity levels were rare when small changes were made to other brain structure activity levels. However, the PVN and raphe nuclei did demonstrate some wide fluctuations in the face of small changes in activity levels. This observation suggests that some further modification of the simulation may be necessary to ensure stability.

Overall, the simulation results are consistent with the serotonin hypothesis based on published neurochemical evidence. Successive increases in the raphe nuclei activity level to $125 \%$ and $150 \%$ led to respective decreases in the activity level of PVN to $75 \%$ and $1 \%$.

If this were a human patient with anorexia, direct intervention would be imperative. But what kind of intervention? In a treatment based upon changing the activity levels of different brain structures (a possible pharmacological approach), what brain structures would one try to change in order to increase PVN activity so that eating would increase?

One approach might be to directly increase PVN activity. The simulation results of this approach are displayed in Figure 3. PVN activity was arbitrarily set at $100 \%$, or normal functioning. Interestingly, because of multiple brain structure activity, this intervention failed to increase PVN activity. Similar results were observed when PVN activity was increased to $150 \%$ of normal function.

Clearly, another approach is required. Since the VTA excites the CN, which in turn inhibits the PVN, decreasing VTA activity may result in an increase in PVN activity. VTA activity was decreased to $75 \%$ of normal. This simulation, which is graphically depicted in Figure 4, results in an increase of PVN activity to normal functioning. The finding that the functional level of the PVN increased when the serotonergic output of the raphe nuclei decreased is consistent with Fava et al. (1989), who reported that underweight anorexic patients had CSF levels of the serotonin metabolite 5-HIAA that were $20 \%$ lower than normal.

It is difficult to produce a simulation model that is able to model completely the complex neurochemical and neuroanatomical connections within the human brain. A number of obstacles are encountered in developing reliable simulations of complex systems such as the brain. In our case, the complexity of the functional neuroanantomy of eating and gaps in the knowledge we have about these systems all serve as stumbling blocks to a precise and detailed model. However, as new advances in neuroscience and technology occur, revisions to the model can bring it closer to resembling human brain function. The present model provides a method for examining how complex interactions in the brain influence human behavior.

The use of computer simulations has practical implications in the understanding and treatment of complex behavioral disorders such as anorexia. For example, a computer simulation, such as the one presented here, could be used to predict the action of such drugs as serotonin antagonists or agonists on relevant brain structures and on subsequent eating behavior. The use of computer simulations to predict the outcome of pharmacological studies could precede the use of drugs on human subjects and reduce the number of clinical trials. Computer simulations could also be used to predict the results of other types of research. Experimental lesion studies on animals could be diminished if one could first make a detailed, reliable prediction with the aid of a computer simulation.

As with any research approach, the use of computer simulations has advantages and disadvantages (Lewandowsky, 1993). For one thing, simplifying assumptions must be made to run simulations. In our simulation, these assumptions include the selection of some brain structures and not others, and the simple addition and subtraction of excitation and inhibition effects. These assumptions may be correct but may also be wrong or oversimplified and may limit the usefulness of the simulation in modeling real brain function. At the same time, even simple dynamic simulations such as this one yield intriguing results. Although these results might have been predicted by careful "thought experiments," the dynamic nature of the simulation often produces outcomes that are not easily predicted. If simulation results 
match empirical findings from the living brain, they may contribute to improved treatment strategies and improved understanding of how the human brain works.

\section{REFERENCES}

Aoyagi, K., Oomaru, Y, \& Shimizu, N. (1992). Concurrent measurement of serotonin metabolism and single neuron activity changes in the lateral hypothalamus of freely behaving rat. Behavioural Brain Research, 49, 205-212.

Blundell, J. (1991). Pharmacological approaches to appetite suppression. Trends in Pharmacological Science, 12, 147-157.

BоOCH, G. (1991). Object-oriented design with applications. Redwood City, CA: Benjamin/Cummings.

Carlson, N. R. (1991). Physiology of behavior (4th ed.). Needham Heights, MA: Allyn and Bacon.

Fava, M., Copeland, P. M., Schweiger, M. D., \& Herzog, D. B. (1989). Neurochemical abnormalities of anorexia nervosa and bulimia nervosa. American Journal of Psychiatry, 146, 963-971.

Goldbloom, D. S., Hicks, L. K., \& GarfinKel, P. E. (1990a). Platelet serotonin uptake in bulimia nervosa. Biological Psychiatry, 28, 644647.

Goldbloom, D. S., Hicks, L. K., \& Garfinkel, P. E. (1990b). The serotonin hypothesis of bulimia nervosa: Theory evidence. Canadian Journal of Psychiatry, 35, 741-744.

Jimerson, D. C., Lesem, M. D., HegG, A. P., \& Brewerton, T. D. (1990). Serotonin in human eating disorders. Annals of the New York Academy of Sciences, 600, 532-544.

KAYe, W. H., Ebert, M. H., Gwirtsman, H. E., \& Weiss, S. R. (1984).
Differences in brain serotonergic metabolism between nonbulimic and bulimic patients with anorexia nervosa. American Journal of Psychiatry, 141, 1598-1601

Kaye, W. H., Gwirtsman, H. E., George, D. T., \& Ebert, M. H. (1991). Altered serotonin activity in anorexia nervosa after longterm weight restoration. Archives of General Psychiatry, 48, 556562.

LEWANDOWSKY, S. (1993). The rewards and hazards of computer simulations. Psychological Science, 4, 236-243.

Love, T. (1993). Object lessons. New York: Sigs Books.

MoRLEY, J. E., \& BLUNDELL, J. E. (1988). The neurobiological basis of eating disorders: Some formulations. Biological Psychiatry, 23, 53-78.

Oliveros, S. C., Iruela, L. M., Caballero, L., \& Baca, E. (1992). Fluoxetine-induced anorexia in a bulimia patient. American Journal of Psychiatry, 149, 1113-1114.

Pazos, A., Probst, A., \& Palacios, J. M. (1987). Serotonin receptors in the human brain, III. Autoradiographic mapping of serotonin-1 receptors. Neuroscience, 21, 97-122.

ReYNolds, D. V., \& Del DotTo, J. (1991). An object-oriented approach to brain/behavior relationships. San Francisco: American Psychological Association.

WALSH, B. T., \& DeVLIN, M. J. (1992). The pharmacologic treatment of eating disorders. Pediatric Psychopharmacology, 15, 149-160.

Whicker, M. L., \& Sigelman, L. (1991). Computer simulation applications: An introduction. Newbury Park, CA: Sage.

(Manuscript received November 21, 1994; revision accepted for publication February 1, 1995.) 